\title{
Rare amyloidoma of the tongue base: A case report and review of the literature
}

\author{
GABRIELA MUSAT $^{1}$, ANCA EVSEI ${ }^{2}$, DANIELA CALINA $^{3}$, ANCA OANA DOCEA ${ }^{4}$, SOTIRIOS G. DOUKAS ${ }^{5,6}$, \\ DIMITRA P. VAGELI ${ }^{7}$, CHARITINI NEPKA $^{8}$, DEMETRIOS A. SPANDIDOS ${ }^{9}$ and MIHAELA MITROI ${ }^{10}$
}

\author{
${ }^{1}$ Department of Otorhinolaryngology, University of Medicine and Pharmacy 'Carol Davila', 050474 Bucharest; \\ ${ }^{2}$ Laboratory of Anatomy-Histology, 'Saint Mary' Clinic Hospital, 011172 Bucharest; Departments of ${ }^{3}$ Clinical Pharmacy, \\ and ${ }^{4}$ Toxicology, University of Medicine and Pharmacy of Craiova, 200349 Craiova, Romania; ${ }^{5}$ Department of Internal Medicine, \\ Saint Peter's University Hospital, New Brunswick, NJ 08901, USA; ${ }^{6}$ Department of Forensic Sciences and Laboratory of Toxicology, \\ School of Medicine, University of Crete, 71003 Heraklion; ${ }^{7}$ Department of Surgery, The Yale Larynx Laboratory, \\ New Haven, CT 06510, USA; ${ }^{8}$ Department of Cytopathology, University Hospital of Larissa, 41110 Larissa; \\ ${ }^{9}$ Laboratory of Clinical Virology, School of Medicine, University of Crete, 71003 Heraklion, Greece; \\ ${ }^{10}$ Department of Otorhinolaryngology, University of Medicine and Pharmacy of Craiova, 200349 Craiova, Romania
}

Received November 29, 2019; Accepted January 8, 2020

DOI: $10.3892 / \operatorname{mco} .2020 .1972$

\begin{abstract}
Localized amyloidosis is a rare condition characterized by the deposition of misfolding protein in a tissue, without other systemic manifestations. Only a small number of cases of localized amyloidosis of the tongue have been reported to date, in contrast to systemic amyloidosis, in which localization on the tongue is common. This study presents a rare case of localized amyloidosis of the tongue (amyloidoma) and provides a summary of the known literature of localized amyloidosis. This study describes the case of a 36-year-old female who presented with a swelling of the tongue base. The diagnosis of amyloidoma was made based on the findings of the physical examination, head and neck MRI findings and the histopathological examination with Congo red stain under polarized light. The histopathological diagnosis was as follows: Localized lambda light-chain amyloidosis. A thorough physical examination was performed by the ENT and Hematology/Oncology departments, without revealing signs of systemic disease. A series of hematological and imaging tests were also performed to verify that there was no sign of systemic involvement. The patient declined surgical excision
\end{abstract}

Correspondence to: Dr Daniela Calina, Department of Clinical Pharmacy, University of Medicine and Pharmacy of Craiova, 2 Petru Rareş Street, 200349 Craiova, Romania

E-mail: calinadaniela@gmail.com

Dr Anca Oana Docea, Department of Toxicology, University of Medicine and Pharmacy of Craiova, 2 Petru Rareş Street, 200349 Craiova, Romania

E-mail: ancadocea@gmail.com

Key words: amyloidoma, rare condition, tongue, biopsy, microscopic examination, immunohistochemistry testing and the 2-year follow-up did not reveal any changes in tumor dimension. Although the etiology of localized amyloidosis is yet not clear, the prolonged reaction of tissue plasma cells to environmental antigens may be a causative factor for the initiation of the neoplastic process.

\section{Introduction}

Amyloidosis is a rare progressive disorder that includes a group of diseases characterized by accumulation of pathologic deposits of amyloids in the tissues. The amyloids are protein polymers formed of identical monomer units. Pathological amyloids are usually formed from misfolded proteins. The deposits of amyloids situated either intracellularly or extracellularly alter the normal function of organs. There are over 23 proteins that can form amyloid fibers in vivo (1).

There are two main types of amyloidosis, namely systemic and localized. Localized amyloidosis is rare, and it usually occurs in the head and neck region, mostly in the larynx and trachea $(2,3)$. Tongue involvement is common in systemic amyloidosis and can be diffuse as macroglossia or localized (4-7). Localized tongue amyloidosis is extremely rare. This study presents a rare case of localized amyloidosis at the level of the tongue base and also reviews the important aspects of the literature for localized amyloidosis.

\section{Case report}

A 36-year-old female with a history of dysmenorrhea and menorrhagia due to uterine fibroids was initially admitted for hysterectomy in the 'Queen Mary' Hospital of Bucharest, Romania. Her complaint was dysmenorrhea and menometrorrhagia. Uterine ultrasound was consistent with the diagnosis of uterine fibroid dimensions 45/6/44 $\mathrm{mm}$. The gynecologists did not perform the surgery. The intervention was terminated due to difficult intubation. A biopsy of the uterine lesions was not 
available. During the intubation attempt, a right-sided tongue tumor was noted, thus rendering intubation difficult and the hysterectomy was thus aborted. Subsequently, the patient was referred to the Ear Nose Throat (ENT) Department of 'Queen Mary' Hospital of Bucharest for further evaluation.

At presentation, the patient did not report dyspnea, dysphonia, dysphagia, hemoptysis, or dysarthria. The patient did not report weight loss, fever, chills, joint pain, skin changes, rashes, numbness or paresthesia. An analysis of her past medical history revealed pulmonary tuberculosis treated 10 years prior (she had completed anti-TB treatment and had a complete recovery), hypercholesterolemia, gastric ulcer and uterine fibroid. A physical examination revealed a yellowish mass on the tongue base, on the right side. The mass was non-friable and soft in nature. A flexible endoscopy was performed, revealing a diameter of approximately $2-3 \mathrm{~cm}$ and a thickness of approximately $1 \mathrm{~cm}$ that occupied the right vallecula (Fig. 1).

A head and neck MRI with and without contrast revealed a polypoid protrusive mass with a length/width/depth of $20 / 7 / 17.8 \mathrm{~mm}$ at the base of the tongue, on the right, partially occupying the right vallecula without evident signs of invasion (Fig. 2). A biopsy of the tumor was performed using a biopsy clamp and several bioptic fragments from the tumor were obtained.

A gross examination revealed multiple tissue fragments with variable dimensions ranging from $0.5 / 0.5 / 0.3 \mathrm{~cm}$ and 0.5/0.5/0.5 cm, with a waxy, starch-like, translucent and firm consistency. A microscopic examination following hematoxylin and eosin staining revealed subepithelial deposits of an acellular, extracellular, eosinophilic and homogenous matrix material; a sparse inflammatory infiltrate composed of lymphocytes and plasma cells was identified. Congo red staining was performed, revealing red homogenous subepithelial deposits (Fig. 3). Red homogenous subepithelial deposits turn into an apple-green birefringence under polarized light (magnification, $\mathrm{x} 40$ and $\mathrm{x} 4$ ) (Fig. 4). Immunohistochemical staining was positive for CD20, indicating B lymphocytes and light-chain lambda amyloid deposits, and was negative for amyloid deposits.

The consulting hematologist performed a thorough evaluation of the amyloidosis, including a chest X-ray, 2D echocardiogram, NT-pro-brain natriuretic peptide, liver fibroscan, immunoelectrophoresis and the Schirmer test. The results of all these tests were within normal ranges, without any signs to suggest systemic disease. The patient refused to undergo fat tissue biopsy.

\section{Discussion}

The classification of amyloidosis is often based on clinicopathological criteria. In this scheme i) primary amyloidosis is systemic, but has no identifiable cause (Table I); ii) secondary amyloidosis is systemic and is often caused by a chronic disease, such as tuberculosis or rheumatoid arthritis; iii) amyloidosis associated with multiple myeloma is multisystemic; and iv) localized amyloidosis is characterized by a lack of evidence of systemic amyloidosis or underlying chronic disease. It is noteworthy that a number of cases with localized amyloidosis have been associated with Sjögren's syndrome $(8,9)$.

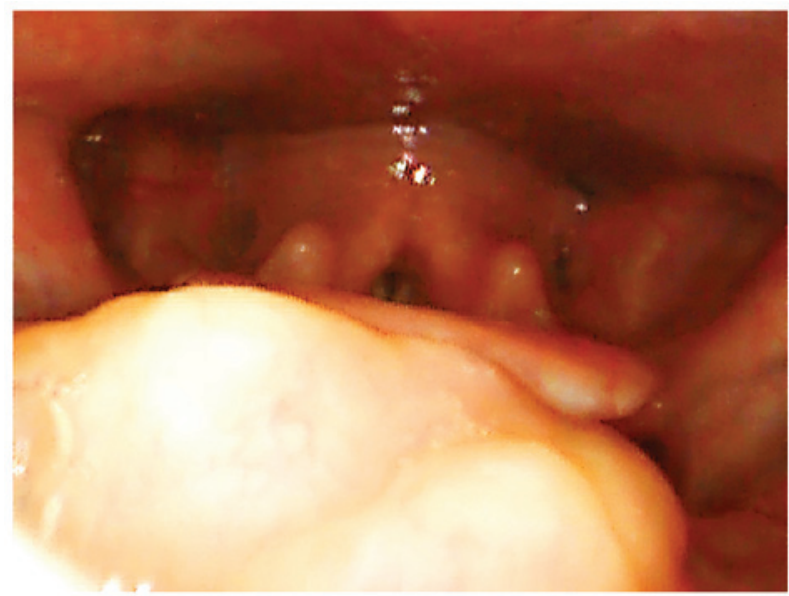

Figure 1. Endoscopic view showing a yellowish mass at the base of the tongue on the right side. The epiglottis is partially visible, on the left side, as well as the arytenoids.
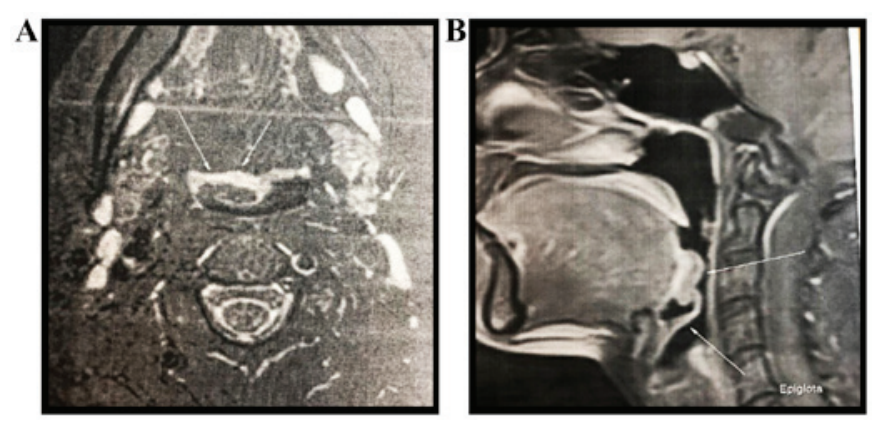

Figure 2. (A) MRI axial and (B) sagittal view showing a polypoid mass located on the base of the tongue, partially occupying the right vallecula. Arrows indicate the lining between tumor and normal tissue (lack of invasion)
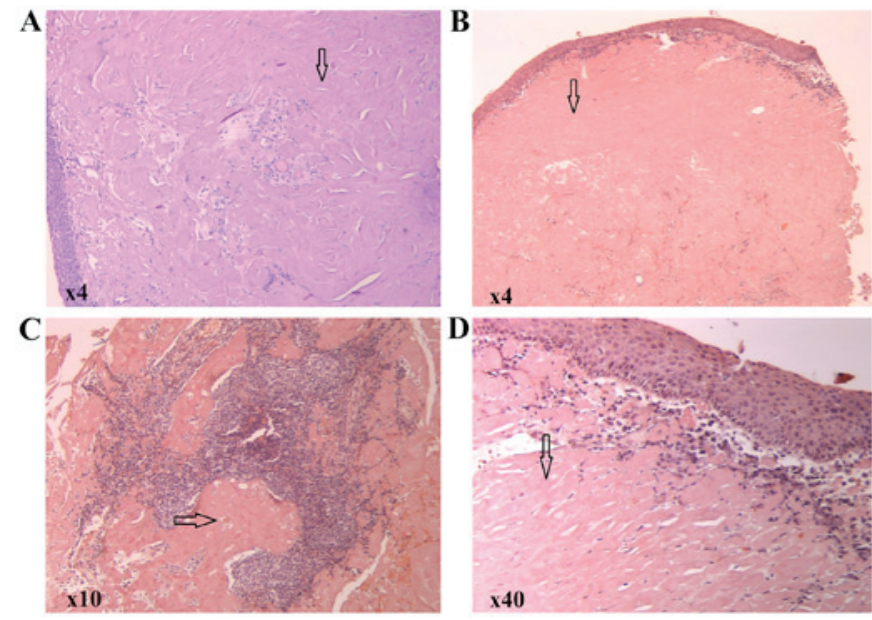

Figure 3. (A) Hematoxylin and eosin staining showing an eosinophilic amorphous material in the connective tissue beneath the intact epithelium (magnification, $x 4$ ). (B-D) Congo red staining showing a red homogenous material (arrows) under light microscopy (magnification, x4, x10 and x40, respectively).

The modern classification of amyloidosis is chemical and is based on the type of protein that is deposited and characterized as AA or AL amyloidosis. 'A' stands for amyloid, followed by 
Table I. Tests used to rule out systemic amyloidosis.

\begin{tabular}{ll}
\hline Tests & \multicolumn{1}{c}{ Results } \\
\hline Chest radiograph & Normal \\
Echocardiography & Normal \\
NT pro BNP & In normal range \\
Liver fibroscan & Within normal limits \\
Basic laboratory investigation & Unremarkable \\
Unoelectrophoresis & Within normal limits \\
Schirmer & Normal
\end{tabular}

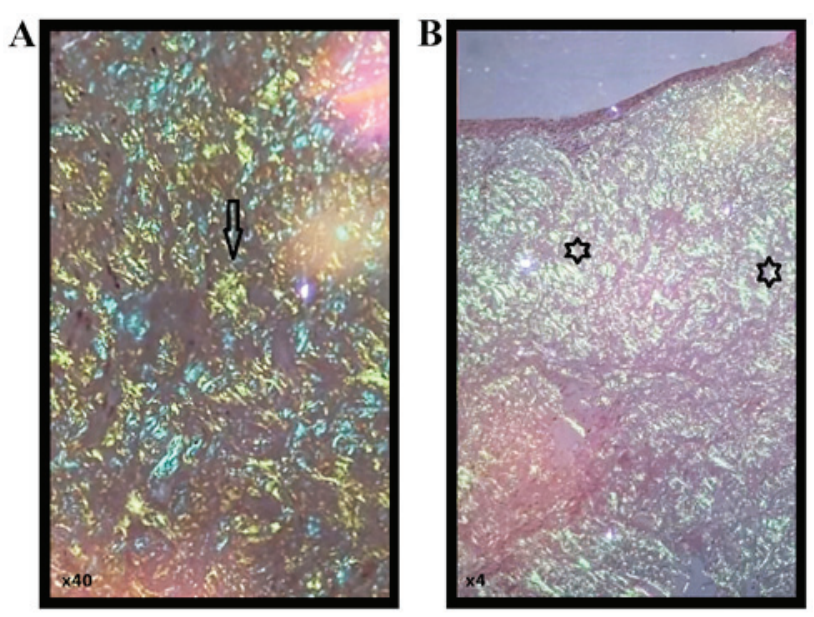

Figure 4. Red homogenous subepithelial deposits (star symbols) in Congo red staining ( $\mathrm{A}$ and $\mathrm{B}$ ) turn into an apple-green birefringence (arrows) under polarized light [magnification, $\mathrm{x} 40$ (A) and $\mathrm{x} 4$ (B), respectively].

the fibril protein abbreviation. For example, light-chain fibril proteins are abbreviated as ' $\mathrm{L}$ '; thus, AL amyloidosis indicates light-chain amyloidosis (10). AL amyloidosis occurs when the bone marrow produces abnormal antibodies that cannot be broken down. The antibodies are deposited in the tissues as amyloid, interfering with normal functions. AA indicates serum A amyloidosis.

Systemic amyloidosis is determined by different types of amyloid deposits, as follows: i) AA amyloidosis, which has as a precursor, the serum amyloid $\mathrm{A}$, is the most frequent systemic amyloidosis and it usually involves the liver, kidneys and spleen (11). It is implicated in various rheumatological disorders, hematological malignancies, autoimmune disorders, hematological malignancies (Hodgkin's disease and non-Hodgkin's lymphoma and multiple myeloma), rheumatologic diseases (rheumatoid arthritis, ankylosing spondylitis and juvenile idiopathic arthritis), autoimmune disorders (psoriasis and psoriatic arthritis). Previously-treated tuberculosis and chronic inflammation due to recurrent respiratory tract infections were considered to be responsible for AA amyloidosis (12). ii) AL (light-chain) amyloidosis is another type of systemicamyloidosis, in which the precursor protein is a clonal immunoglobulin light-chain or light-chain fragment. The organs involved are usually the heart, kidneys, gastrointestinal tract, respiratory tract and peripheral nervous system. iii) $\mathrm{AH}$ amyloidosis is produced by the fibrils of heavy chain sequences of immunoglobulin. There are also transthyretin amyloidosis (ATTR), beta 2 microglobulin amyloidosis (Abeta2M) and other types of systemic amyloidosis.

Localized amyloidosis is formed by the local production and deposit of fibers in different tissues. In localized amyloidosis, plasma cells secrete light-chain immunoglobulins that misfold and are deposited locally (13-15). Localized AL amyloidosis ('amyloidoma') represents a true plasma cell neoplasm and not a pseudotumor. Specifically, although localized AL amyloidosis appears as a tumor according to a classical definition, the basic lesion is a neoplasm in the form of a clonal expansion of one plasma cell (3). The sites of development of localized amyloidosis include the bladder, trachea, bronchi, larynx, gastrointestinal tract, orbit, tonsils, lymph nodes and skin. One of the most well-studied localized types of amyloidosis is the one localized in the brain tissue typical in Alzheimer's disease (16).

The exact etiology of localized amyloidosis is not yet known. Given that the amyloidosis is usually formed in mucous membranes, it has been suggested that the prolonged reaction of tissue plasma cells to environmental antigen is the first step (17). These reactive cells exhibit an increase production of immunoglobulin light-chains that are amyloidogenic. The transformation of these immunoglobulin light chains to insoluble fibrils by macrophages seems to be a key step in the formation of amyloidoma. This theory is supported by the fact that giant cells are a common finding in cases of localized amyloidoma (3).

The larynx is the most involved organ in head and neck localized amyloidosis. In contrast to systemic amyloidosis in which tongue involvement is common, leading to extensive enlargement of the tongue known as macroglossia (5), the tongue is seldom a place of appearance for the localized amyloidosis and a small number of cases has been reported (7) and can be expressed as nodules of local accumulation of protein deposits (18). The most extensive case series has been published by the Mayo Clinic in 2013; they reported 6 cases of localized tongue amyloidosis in a 42-year period of time (7).

The clinical suspicion of amyloidosis requires histological confirmation (19). The majority of localized oral amyloidoses are easily biopsied under local anesthesia. Tissue biopsy and microscopic examination is usually sufficient to establish a diagnosis. The amyloid stained with hematoxylin and eosin staining in light microscopy, appears as a homogenous eosinophilic, amorphous substance. However, following staining with Congo red and placed under polarized light, it reveals an apple-green birefringence. If the result is positive, the next step would be to establish the subtype of amyloidosis. This is usually tested using serum or urine immunofixation electrophoresis to search for a clonal disorder. In the case of a negative result, immunohistochemistry is useful in finding $\mathrm{K}$ or lamda light-chains to confirm a clonal disorder - AL and amyloid A to confirm AA. Nevertheless, this type of testing is neither specific nor precise. Future studies should be performed on a larger cohort of patients for more reliable results (20).

Oral localized amyloidosis can easily be found on the tongue, which can be enlarged diffusely due to macroglossia or as nodular deposits (21). If the disorder is extensive, the tongue usually loses its elasticity and may be firm, fissured, ulcer- 
ated and occasionally red and painful. Function impairment is present, interfering with speech, chewing and swallowing. Macroglossia is rare in type AA and more frequent in type AL, although associating the type of amyloid to the type of clinical presentation is not recommended. Tissue biopsy is mandatory for histological confirmation.

Current recommendations regarding amyloidosis diagnosis are the following: i) Congo red stain is currently the gold standard for amyloid detection; ii) the type of amyloid must be identified microscopically or immunohistochemically, not solely on clinical or DNA studies; iii) immunohistochemistry has to be performed cautiously and amyloidosis suspicion must be completed using more sophisticated methods in referral centers (22).

The prognosis for patients with AL amyloidosis is linked to the extent of the disease. Localized amyloidosis has an excellent prognosis and rarely progresses to systemic disease. A study on 606 cases of localized amyloidosis over a period of 30 years revealed that only one case progressed to a systemic AL (4,23). Therefore, patients with localized form usually do not require treatment with systemic therapy. Management can be supportive or localized. The most common approaches include local excision, ablation or clinical observation. Surgical excision or ablation of the amyloidoma is usually the treatment of choice and is indicated for symptomatic management to ensure the airway patency; however, recurrence is frequent and revisions may be required (24).

Tongue amyloidoma is a rare presentation of localized amyloidosis. A limited number of cases of localized amyloidosis have been previously reported to involve the tongue $(2,3)$, in contrast to systemic amyloidosis that can commonly involve the tongue (4-7).

This study presents a rare localized amyloidoma of the tongue. Taking the history into consideration, physical findings, head and neck MRI findings and histopathological results, using Congo red stain, the diagnosis of localized lambda light-chain amyloidosis of the tongue base was made. Although, active TB is associated with systemic amyloidosis $(2,12)$, as the patient had a complete recovery, and investigations did not reveal any signs of systemic amyloidosis, the amyloidosis was considered localized.

Usually, localized amyloidosis does not progress to systemic involvement and the prognosis is excellent. Surgical resection is the treatment of choice; however, clinical follow-up can also be performed if the airway is not compromised. In the current case, the therapeutic plan presented herein was initially the removal of the tumor by laser or electrocautery, in suspension laryngoscopy; however, the patient refused. The pros and cons of the procedure were extensively discussed with the patient. The patient refused treatment at this point and follow-up for further clinical monitoring was arranged. The 2-year follow up of the patient did not reveal any change in the size of the tumor, or further symptomatology.

In summary, i) the cases of localized amyloidosis of the tongue remain rare; ii) the diagnosis of systemic examination should be evidence based and necessary investigations to rule out systemic causes of amyloidosis; iii) although the localized amyloidomas may have a localized mass effect, prognosis is overall good; and iv) the etiopathogenesis of localized amyloidosis is not yet clear and further investigations are required.

\section{Acknowledgements}

Not applicable.

\section{Funding}

No funding was received.

\section{Availability of data and materials}

All data generated or analyzed during the current study are included in this published article or are available from the corresponding author upon reasonable request.

\section{Authors' contributions}

All the authors (GM, AE, DE, AOD, SGD, DPV, CN, DAS and $\mathrm{MM}$ ) were involved in conceiving and designing the study. GM, AE and MM contributed to sample collection. DC, AOD, SGD and DPV performed the statistical analysis. DC, AOD, GM, SD, DPV and MM drafted and wrote the manuscript. AOD, DC, DAS and CN gave advice on the experimental design, interpreted the results and critically revised the manuscript. All authors have read and approved the final version of the manuscript.

\section{Ethics approval and consent to participate}

The access of the database for the purpose of this study was approved by the Ethics Committee of 'Saint Mary' Clinic Hospital, Bucharest, Romania. As the hospital to which the patient was admitted is a teaching hospital, all patients admitted to our hospital signed a written consent by which they agree that their medical data can be used in scientific studies.

\section{Patient consent for publication}

The participation in the study was approved by the patient and she gave her approval for the patient information presented herein to be published.

\section{Competing interests}

DAS is the Editor-in-Chief for the journal, but had no personal involvement in the reviewing process, or any influence in terms of adjudicating on the final decision, for this article. All the other authors declare that they have no competing interests.

\section{References}

1. Buxbaum JN: The systemic amyloidoses. Curr Opin Rheumatol 16: 67-75, 2004.

2. Kerner MM, Wang MB, Angier G, Calcaterra TC and Ward PH: Amyloidosis of the head and neck. A clinicopathologic study of the UCLA experience, 1955-1991. Arch Otolaryngol Head Neck Surg 121: 778-782, 1995.

3. Westermark P: Localized AL amyloidosis: A suicidal neoplasm? Ups J Med Sci 117: 244-250, 2012

4. Mahmood S, Sachchithanantham S, Bridoux F, Lane T, Rannigan L, Foard D, Sayed R, Patel K, Fontanna M, Whelan C, et al: Risk of Progression of localisedamyloidosis to systemic disease in 606 patients over 30 years. Blood 122: 3143, 2013. 
5. van der Wal N, Henzen-Logmans S, van der Kwast WA and van der Waal I: Amyloidosis of the tongue: A clinical and postmortem study. J Oral Pathol 13: 632-639, 1984.

6. Pentenero M, Davico Bonino L, Tomasini C, Conrotto D and Gandolfo S: Localised oral amyloidosis of the palate. Amyloid 13: 42-46, 2006

7. O'Reilly A, D'Souza A, Lust J and Price D: Localized tongue amyloidosis: A single institutional case series. Otolaryngol Head Neck Surg 149: 240-244, 2013

8. Moon AO, Calamia KT and Walsh JS: Nodular amyloidosis: Review and long-term follow-up of 16 cases. Arch Dermatol 139: 1157-1159, 2003.

9. Biewend ML, Menke DM and Calamia KT: The spectrum of localized amyloidosis: A case series of 20 patients and review of the literature. Amyloid 13: 135-142, 2006.

10. Westermark P, Benson MD, Buxbaum JN, Cohen AS Frangione B, Ikeda SI, Masters CL, Merlini G, Saraiva MJ and Sipe JD: A primer of amyloid nomenclature. Amyloid 14 179-183, 2007

11. Hazenberg BP: Amyloidosis: A clinical overview. Rheum Dis Clin North Am 39: 323-345, 2013.

12. Balwani MR, Kute VB, Shah PR, Wakhare P and Trivedi HL: Secondary renal amyloidosis in a patient of pulmonary tuberculosis and common variable immunodeficiency. J Nephropharmacol 4: 69-71, 2015.

13. Berk JL, O'Regan A and Skinner M: Pulmonary and tracheobronchial amyloidosis. Semin Respir Crit Care Med 23: 155-165, 2002.

14. Charlot M, Seldin DC, O'Hara C, Skinner M and Sanchorawala V: Localised amyloidosis of the breast: a case series. Amyloid 18 : 72-75, 2011.

15. O'Regan A, Fenlon HM, Beamis JF Jr, Steele MP, Skinner M and Berk JL: Tracheobronchial amyloidosis. The Boston University experience from 1984 to 1999. Medicine (Baltimore) 79: 69-79, 2000 .
16. Hardy J and Selkoe DJ: The amyloid hypothesis of Alzheimer's disease: Progress and problems on the road to therapeutics. Science 297: 353-356, 2002

17. Krishnan J, Chu WS, Elrod JP and Frizzera G: Tumoral presentation of amyloidosis (amyloidomas) in soft tissues. A report of 14 cases. Am J Clin Pathol 100: 135-144, 1993.

18. Kubota K, Ito R, Furudate K, Kon T, Nakagawa $H$ and Kobayashi W: Localized AL amyloidosis of the tongue: A case report and literature review. J Oral Maxillofac Surg Med Pathol 29: 142-145, 2017.

19. Thompson L and Bishop J: Head and Neck Pathology. 3rd edition. A Volume in the Series: Foundations in Diagnostic Pathology. Elsevier, 2019.

20. Binmadi N, Intapa C, Chaisuparat R, Akeel S, Sindi A and Meiller T: Immunophenotyping Oral Amyloidosis for the Precise Identification of the Biochemical Forms: A Retrospective Study. Open Dent J 12: 1036-1042, 2018.

21. Picken MM: Amyloidosis-where are we now and where are we heading? Arch Pathol Lab Med 134: 545-551, 2010.

22. Yamagata $\mathrm{K}$ and Bukawa $\mathrm{H}$ : Oral localised amyloidosis. In: Amyloidosis - An Insight to Disease of Systems and Novel Therapies. IntechOpen, London, 2011. https://www.intechopen.com/books/ amyloidosis-an-insight-to-disease-of-systems-and-novel-therapies/ oral-localized-amyloidosis. Accessed November 16, 2011

23. Girnius S: Overview of systemic and localised amyloidosis. Rev Health Care 4: 231-247, 2013.

24. Wald MH: Clinical studies of secondary amyloidosis in tuberculosis. Ann Intern Med 43: 383-395, 1955.

This work is licensed under a Creative Commons Attribution-NonCommercial-NoDerivatives 4.0 International (CC BY-NC-ND 4.0) License. 